\title{
Facial Comparisons by Subject Matter Experts: Their Role in Biometrics and Their Training
}

\author{
Nicole A. Spaun \\ Federal Bureau of Investigation \\ Forensic Audio, Video and Image Analysis Unit \\ Building 27958-A, Pod E, Quantico VA 22135 USA, 703-985-1169 \\ Nicole.Spaunaic.FBI.gov
}

\begin{abstract}
The fingerprint biometrics community has accepted the involvement of human examiners as the ultimate verifiers of the output of automated systems, especially in latent fingerprint cases. Likewise, the facial biometrics community should recognize the importance of human experts working in association with the automated process, particularly when analyzing uncontrolled images. As facial biometric systems become more common, more facial image examiners will need to be trained. Currently there are no systematic training programs in place for these examiners. This paper outlines the knowledge needed to conduct facial examinations, and thus should be taught in a successful training program. A facial image comparison expert must be versed in many subjects including: comparative science, image science and processing, bones of the head, muscles of the face, properties of the skin, aging and alteration, legal issues and case law, and the history of facial identifications and photographic comparisons.
\end{abstract}

Keywords: Biometrics, forensics, facial recognition, facial identification.

\section{Introduction}

When automated fingerprint comparison systems, such as IAFIS, went into service, there were numerous human experts readily available to verify the results of those programs. In the United States, tens of state and local law enforcement agencies employ fingerprint examiners; this is in addition to the hundreds of experts employed within the US Departments of Justice, Defense, and Homeland Security alone. In contrast, there are comparatively few trained subject matter experts currently practicing facial comparisons for identification in justice systems around the world. Within the United States, there are less than 10 federal, state, and local agencies combined practicing forensic facial comparison today. Many agencies are welcoming the introduction of automated facial recognition systems as an answer to the comparison of people from surveillance images; however these agencies generally lack the experts to verify those results. As such, automated facial recognition will necessitate a substantial increase in the number of facial image examiners worldwide. Because there are currently no automated facial biometric systems with high enough accuracy to allow for "lights out" operation, forensic examiners will need to verify the resultant output 
of these systems. Therefore, training and certification programs for facial image examiners need to be developed today for the coming wave of automated facial biometrics tomorrow.

This paper presents an outline of the history of facial identification in the legal system, the role for human subject matter experts in the automated facial recognition cycle, an outline of the levels of expertise needed to perform facial identifications for various purposes, and a summary of the spheres of knowledge that a facial image examiner would need to be versed in to perform scientific comparisons of faces.

\subsection{History of Facial Identification, or Why There Are Few Trained Experts Today}

The history of identification of people by their facial morphology in the legal system is a long and often punctuated one. In the late 1800's, Parisian anthropologist Alphonse Bertillon established a system for personal identification based on measurements of the face and body. Concurrently, fingerprinting was gaining popularity in India and England to identify people in the criminal justice system. At the turn of the century, the Bertillon system of identification fell out of favour when the uniqueness of the selected measurements was challenged by instances of misidentification [1]. Subsequently, fingerprinting quickly replaced the Bertillon system as the dominant means of identifying people to the exclusion of all others. The need for facial identification subject matter experts waned. The comparison of faces for official purposes was left to police, soldiers, guards, and other individuals, most of whom received more training in firearms than faces, if at all. As photography and surveillance became more prevalent through the $20^{\text {th }}$ century, a small number of agencies began developing specialists to compare photographs of the faces of individuals. For example, during the Cold War the US Central Intelligence Agency compared photographs to identify Communist officials and their look-alikes [2].

Facial identification has suffered perception problems. While few people challenge the notion that experts are needed to compare fingerprints or footwear patterns to draw a proper conclusion, the majority of people believe that anyone can accurately identify faces in images without training and therefore experts are not needed. In the 1970's, Federal Bureau of Investigation (FBI) photographic examiners testified in several cases where the courts proposed that the conclusions of the facial comparison experts, while not harmful, may not be necessary except in the most difficult cases as the jury can view the images themselves and draw their own conclusions [3]. With such case law as precedent, it is apparent why few other agencies invested in establishing and training experts. Fortunately, subsequent cases have stated that subject matter expert testimony concerning facial comparisons is indeed beneficial.

Because people are accustomed to recognizing faces and not accustomed to comparing firearms, footwear, or fingerprints, it is assumed that experts are needed for analyzing the latter. However, the ability to conclusively identify people in images is different than recognizing individuals in person. Images are two-dimensional representations of a scene; while faces are highly three-dimensional involving many variables, including optics and perspective, that factor into facial image analysis. Thus it is the role of the facial image examiner to draw a scientific conclusion from the comparison of faces, acknowledging the complex variables, and their effects, in the process of imaging and comparing the human face. 


\subsection{The Current State of Forensic Facial Identifications}

Unlike in fingerprint biometrics where the community developing automated systems realized the significant role that human experts will play in the course of action integrating with the automated systems, the facial biometric community is currently focused on developing new algorithms or attempting to fuse biometrics. The simple solution to enhance the accuracy of automated systems right now is to utilize human subject matter experts in the procedure. Also, it is essential that the output of automated facial recognition systems is verified by human examiners if it is to be used for legal purposes, just as is done with latent fingerprints. The availability of these subject matter experts, however, is an issue at the present time.

Currently there are numerous agencies using automated facial biometric systems to determine identities for purposes, such as access control and fraud detection, however often the people operating these systems have little or no facial and/or image analysis training. The agencies using these systems have generally created in-house training as external training is rarely available. Therefore, agencies that do have trained facial comparison subject matter experts are besieged with requests for their training programs and expert assistance. As such, today's subject matter experts on facial comparison must ensure the proficiency of the next generation of experts by sharing their training programs and science. A certification in facial image comparisons should be developed for the future and the first step is to define the topics of which an expert should have knowledge and practice.

\section{Levels of Training}

In an ideal world, every person comparing images of faces will be trained to the highest possible level of knowledge and practice in their field. In reality, this is not essential as the comparison of certain images involves fewer variables and, as a result, less training is needed to draw conclusions in such cases. Fingerprint comparison examinations are typically divided into two types: ten-print and latent print. Comparable to the differentiation of fingerprint comparisons by the type/quality of fingerprints under examination, facial comparison examinations can be divided by the type of images depicting faces that will be subject to facial comparison.

The terms controlled and uncontrolled have been used to describe the conditions under which the biometric sample has been obtained [4]. In facial biometrics, controlled images are front facial pictures with a subject bearing a neutral expression who is photographed under controlled, even lighting in front of a neutral background. Recommended standards exist for the taking of controlled images for the purpose of identification, which includes photographs taken for passports and arrest records (e.g. [5]). Uncontrolled images are simply that: not controlled. They are subject to great variations, including the pose and expression of the subject, the lighting and background of the environment, the distance between the camera and subject, etc. Examples of uncontrolled images include snapshots or surveillance images from both video and still cameras. In order for an examiner to understand and account for these complex, uncontrolled variables, they must have knowledge and expertise, and thus training, beyond that of examiners who are solely analyzing controlled, standardized images. Therefore, it can be suggested that examiners performing facial comparisons of 
controlled-to-controlled images are to be trained at a basic level and examiners performing facial comparisons of any uncontrolled images be trained to an advanced level. For the purposes of this paper, examiners trained to a basic level are referred to as Standardized Image Examiners (SIE) and those trained to an Advanced level are referred to as Uncontrolled Image Examiners (UIE) and cumulatively both types together shall be referred to as Facial Image Examiners (FIE).

\section{Spheres of Training}

The areas of training for facial image examiners can be divided into two categories: general and facial specific. An additional training component concerning legal issues and case law is essential for facial image comparison experts working within the justice system and/or are expected to present their conclusions in court. The general knowledge necessary for facial image examiners is similar to that taught for footwear, fingerprint, and other forensic disciplines involving the comparison of items. The science of comparing objects, including those depicted in images, has been documented in other forensic disciplines and therefore facial comparison training should receive similar training. Specifics of the face is a topic that should be covered in detail in any facial image training.

\subsection{General Knowledge}

History. Any training program in facial comparison, or any biometric for that matter, must first include a review of the history of personal identification. By exploring the past of any field, one learns about the advances and relapses that have occurred. A discussion of the early methods of individualizing persons would include an overview of the Bertillon system of personal measurements and a brief history of fingerprinting. Because facial image analysis is a subset of the discipline of Photographic Comparison, the history of the science of photographic comparisons should also be included, with a particular focus on photographic comparisons of faces.

Biometric Advances. An overview of automated facial biometrics should include focus areas such as the operation of common automated systems and the typical output of such automated systems. It is important for all facial image examiners to be familiar with the underlying methods of facial recognition systems, whether they are directly using these systems or not. The advanced level of training should include a brief review of the specific algorithms at use in most systems. Such a discussion would benefit the Uncontrolled Image Examiner by elucidating the sensitivities of these algorithms to the variable factors present in complex surveillance-type images. For example, some algorithms are most sensitive to facial geometry and therefore camera to subject distance variations would greatly affect those programs, while other systems are most affected by the choice of their training set of images.

Underlying Principles. The principles of photographic comparison are those of identification science: individuality and comparison. In the forensic community these principles are commonly referred to as ACE-V [6], which stands for Analyze, Compare, Evaluate, and Verify. Both the principles of individuality and of comparison are critical elements for the facial image examiner to learn. 
The principle of individuality states that no two objects in the universe are the same [6]. Identical twins can be distinguished in images by observing features such as blemishes and scars; twins can also be individualized by ear structures and fingerprints. Because identical twins can be individualized, it can be inferred that any other randomly selected two people can be distinguished. A major challenge in identifying people by their facial morphology and geometry is the lack of statistical analyses available to document the percentage of uniqueness. The lack of specific statistics restricts the facial image examiner within the justice system to opinion based testimony. Automated facial recognition systems generate 'probabilities of match'. Without grounding in the actual statistical uniqueness of faces, however, those percentages can be considered subjective as they depend more on the chosen algorithm than observable traits. For instance, it is common for the true match in the output of a facial recognition system to be in a lower position in the gallery than the first position, implying that the system identified another individual as the best match to the probe. Future research must determine the statistics of facial uniqueness to explain the logic of match probabilities generated by the systems.

The principle of comparison is the scientific methodology for assessing the similarities and differences between items or persons. The ACE-V expression of this principle as applied to forensic science was articulated in 1973 for fingerprint, footwear and tire-tread analyses: to analyze the items, to compare their class and individual characteristics, to evaluate the significance of the similarities and dissimilarities, and to form an accurate conclusion that could be reached by a similarly trained examiner [6]. This method also applies to photographic comparisons of persons. Training in this area should be comprehensive and include hands on exercises to familiarize examiners with the methodology.

Image Science. Because the facial comparison is image based, it is important for facial comparison experts to know basic image science. Controlled facial imaging involves fewer variables than uncontrolled surveillance-type images and therefore the Uncontrolled Image Examiner will need to understand more factors than the Standardized Image Examiner. All examiners should be trained in the properties of digital images and cameras, including the basics of sensors and lenses. Additionally, one should understand compression and the effects it can have on an image. For example, the blocky artifacts created with JPEG compression could mask moles, freckles, and other smaller facial features. Also, understanding image perspective will allow facial image examiners to account for potential differences in facial size and shape; even in controlled images the subject is frequently asked to stand too close to the camera. If a person is less than 4 to 6 feet from a camera, regardless of lens or camera-make used, the perspective will distort the person's face, making their nose appear larger and their ears appear smaller than in reality. Thus it is always preferable to have the subject stand farther away from the camera and use an optical zoom with the camera, than to have a person stand very close to the camera.

Advanced training in image science will include more details about optics. It is not uncommon for surveillance-type images to exhibit barrel distortion, where the outer edge of the depicted scene can appear stretched. Should a face be present in this distortion area, the shape of the face can appear significantly different than in a comparison image. Likewise, uncontrolled images are subject to a wide variety of illumination differences which affect the appearance of the human face. For example, a controlled image should depict freckles on a face, where an uncontrolled image taken with a strong flash may depict a bright area on the person's cheeks, obscuring any facial freckles. 
Image Processing. An important tool in image analysis is image processing. Basic image processing includes brightness and contrast adjustments, as well as rotations and cropping of images. Rotating images can be beneficial to align features, such as making the line of the eyes horizontal, and cropping can remove background information that is distracting to the facial depiction. Training should include these basic techniques; both knowledge of how they work and when to apply them.

Advanced level training should include several additional image processing techniques, such as sharpening and blurring [7]. Another tool is the separation of color channels in color images to detect subtle facial features. Additionally, an image examiner may be assisted by the creation of an overlay of questioned and known images. An overlay allows the examiner to switch between stacked images to ascertain differences in facial morphology and relative dimensions.

\subsection{Facial Specifics}

The human face is effectively an elastic sculpture built upon the foundation of bones and muscles, wrapped in skin, and subject to changes with expression, age, and damage. The specifics that need to be included in a training program are the properties of the face, the alteration of the face, and the methods of comparing faces.

Properties of the Face. Starting from the inside out is a logical way to become familiar with the human face. All facial image examiners should be trained in the structure of the bones and muscles of the head. Training should include a link between external expressions and the internal muscle groups involved in making such expressions, in order for the examiner to compare images depicting differing facial expressions. Furthermore, because wrinkles are typically formed perpendicular to the direction of muscle extension, knowledge of the muscles of the head is important to understanding the aging of the face. The outermost layer of the face, the skin, bears the texture that is most critical in individualizations. Commonly it is the co-location of facial blemishes, marks, and patterns thereof that allow a facial image examiner to identify a person to the exclusion of all others. A medical understanding of dermatology is not necessary at either the basic or advanced level of training, however a working knowledge of the visible features within the skin and their evolution is needed. For example, an examiner does not need to distinguish between a mole or a freckle but should know whether those dark marks will increase in number or fade in contrast through time.

Special attention should be given to the characteristics of the ear. Researchers have noted that they have not found any two ears that are alike in all parts and the ear is also stable throughout adulthood [8]. At a basic level, a facial comparison expert must be familiar with the names and nature of the features of the ear. At an advanced level, the history of ear individualization and ear comparisons should be reviewed. There are decades of research concerning the uniqueness of ears that could benefit a facial image expert analyzing uncontrolled images depicting views of ears.

To synthesize the physical properties of the face, training should include an overview of general facial shapes and the terminology used to describe them. Additionally, advanced training should include a review of known statistics regarding face shape. 
Alteration of the Face. One of the drawbacks to facial biometrics is that the face is not stable over the life of an individual; nevertheless, it is one of the easiest biometrics to obtain. Alteration of the face can be temporary or permanent, to the texture or the structure. An additional type of alteration in facial image comparisons is alteration of the image itself. Facial image examiners must be aware of the possibilities of disguise and deception in imagery.

Aging of a face is the most common alteration depicted. Automated facial biometric systems are challenged by images of an individual taken years apart, therefore an expert verification can significantly improve the accuracy of the system. The aging process involves changes in both the texture and elasticity of the skin. Lifetime use of muscles will be reflected in the location and depth of facial wrinkles and furrows that develop. Many lifestyle factors will affect the aging of the face, including a history of drug usage, cigarette smoking, or weight changes.

Temporary, or transient, alteration of the face is easily achieved by the use of cosmetics and changes to hair. Makeup and cosmetics can mask the appearance of wrinkles and blemishes. Cosmetic medicine, such as the use of Botox, can also temporarily affect facial creases. Changes in length and color of hair are also transient alterations, although the hairline shape tends to remain stable until receding. Changes in facial hair are also common for both men and women. Women frequently alter their eyebrows and eyelashes, while men typically alter their beards and moustaches. Training should include comparisons of individuals with changes in hair and makeup.

Permanent facial alterations can be intentional or unintentional. Unintentional alterations include the formation of scars and the loss of hair. Intentional permanent alterations are can be achieved through plastic/cosmetic surgery. An examiner should be trained in recognizing these alterations to be able to account for such differences within comparisons.

The presence of possible image manipulation is a concern when individuals are allowed to submit their own images, such as visa applications, or within uncontrolled images obtained from unknown or unrestricted sources, such as second- or third-generation videos obtained from terrorist organizations. Training in the detection of image manipulation can be its own substantial training course; nonetheless some basics should be included in a training program for facial image examiners. Standardized image examiners should be able to recognize changes in the orientation of images, such as being flipped left to right or changes in color. When analyzing uncontrolled images, an examiner at the advanced level should be able to test for image changes due to a composite (i.e., "cut and paste"), such as differing perspective, focus, or lighting across an image.

\subsection{Legal Issues}

The legal issues relating to facial comparisons will vary by country and region. However, it is critical for any facial comparison expert who may testify in the judicial system to be aware of the legal issues and case law pertinent to facial comparisons within their region. All facial image examiners involved in the judicial system, i.e. forensic examiners, should be trained in the legal history of photographic comparisons, particularly facial comparisons, to include specific case law that helped, or hurt, the presentation of the science in court. Other legal issues to be covered involve the admissibility of facial comparisons in court. Within the United States, this involves familiarity with 
the Federal Rules of Evidence and specific rulings, such as Frye, Daubert, and Kumho Tire [9]. Lastly, a forensic facial image examiner should be trained in how to testify to their comparisons in court. Training should involve the creation of visual media to assist in presenting the comparison, as well as an overview of approaches to explaining the science to a layman.

\section{Summary}

Until a 'lights out' system can be developed for facial recognition, human examiners are a necessity. Similar to fingerprint examiners, human facial image examiners with sufficient training can be integrated into the facial recognition biometric chain to increase the accuracy and verify the results output from automated systems. Experts analyzing controlled images can be trained to a basic level because there are fewer variables in standardized images, while experts analyzing uncontrolled images must be aware of additional factors, such as variations in lighting, perspective and optics. The development of a successful training program is the first step in creating a certification program that will ensure a set level of knowledge is achieved by all facial image examiners.

\section{References}

1. Rogers, S.: The Personal Identification of Living Individuals, Charles C Thomas, Springfield Illinois (1986)

2. Brugioni, D.: Photo Fakery: The History and Techniques of Photographic Deception and Manipulation. Brassey's, Dulles Virginia (1999)

3. United States v. Cairns, 480 F.2d 927 (6th Cir. 1973); United States v. Brown, 501 F.2d 146 (9th Cir. 1974); United States v. Trejo, 501 F.2d 138 (9th Cir. 1974)

4. Woodward, J., Orlans, N., Higgins, P.: Biometrics. McGraw-Hill Professional, Blacklick Ohio (2003)

5. ANSI/NIST-ITL 1-2000

6. Tuthill, H., George, G.: Individualization: Principles and Procedures in Criminalistics, 2nd edn. Lightning Powder Company, Jacksonville Florida (2002)

7. Scientific Working Group on Imaging Technology (SWGIT), http: / /www. swgit . org

8. van der Lugt, C.: Earprint Identification. Elsevier, Gravenhage (2001)

9. Frye v. United States, 293 F. 1013 (D. C. Cir. 1923); Daubert v. Merrell Dow Pharmaceuticals, Inc., 509 U.S. 579 (1993); Kumho Tire Co. v. Carmichael, 526 U.S. 137, 154 (1999) 